\title{
Outage Analysis of Relay-Assisted BPSK-SIM Based FSO Systems over Strong Atmospheric Turbulence with Pointing Errors
}

\author{
K. Prabu and D. Sriram Kumar
}

\begin{abstract}
Natural turbulence and scintillation are the major challenges in practical deployment of free space optical (FSO) communication systems. In this paper, a parallel relay assisted FSO system is considered to deal with these challenges. This paper investigates the end-to-end performance of the multihop parallel relay assisted FSO system over a strong atmospheric turbulence channel with misalignment fading. The analysis is carried out for system employing decode-and-forward (DF) relays and for strong turbulence channel which can be modeled by the gamma-gamma distribution. A novel closed-form expression for the outage probability is derived. The results are compared with the direct transmission system.
\end{abstract}

Index Terms-Binary phase shift keying subcarrier intensity modulation, free space optics, outage probability, parallel relay, strong atmospheric turbulence.

\section{INTRODUCTION}

Free-space optical (FSO) communication uses light propagating in free space to transmit data for telecommunications or computer networking. FSO is emerging as a captivating alternative to work out the hindrances in the connectivity problems. Unlike conventional $\mathrm{RF} /$ microwave links, FSO communication systems has huge bandwidth, license-free operation, high security, electromagnetic interference immunity, lower deployment cost and time as compared to conventional $\mathrm{RF} /$ microwave links [1]-[4]. The main undignified factor in FSO links is the atmospheric turbulences particularly for long distance communication, more than a few kilometers [5]. Relay assisted FSO system is an effective method to extend coverage and lessen the effects of fading.

The two relays assisted schemes broadly used in FSO systems are serial relaying and parallel relaying. The outage probability of serial and parallel relay assisted FSO for log-normal atmospheric turbulence channel with path loss effects by using both decode-and-forward (DF), amplify-and-forward (AF) with $\mathrm{K}$ relays is analyzed in [6]. The authors in [7] have studied on the end-to-end performance of serial relay FSO system with AF relays over gamma-gamma channels and employing subcarrier differential phase shift keying modulation (DPSK).

The average bit error rate (BER) performance of the DF based cooperative FSO system over gamma-gamma channel using binary phase shift keying (BPSK) based sub-carrier

Manuscript received March 20, 2014; revised May 10, 2014

The authors are with the Department of Electronics and Communication Engineering at National Institute of Technology, Trichy, India (e-mail: nitprabu@gmail.com,srk@nitt.edu). intensity modulation (SIM) is analyzed [8]. In [9], the different DF relay schemes have been introduced to an FSO communication system and their BER performance is investigated in the presence of shot noise, using the photon count method.

To the best of the author's knowledge, the outage performance of the multi-hop relaying over the gamma-gamma channel with pointing error using BPSK-SIM has not yet been investigated. In this paper, the outage probability of the DF based parallel relay assisted FSO system is analyzed over the strong atmospheric channel with pointing error using BPSK-SIM.

The rest of the paper is organized as follows: Section II discusses the system and channel model used. In Section III, the outage performance of relay assisted BPSK-SIM FSO system is discussed. Section IV describes the numerical results with graphical analysis. Finally, concluding remarks are highlighted in Section V.

\section{SyStem AND CHANNEL Model}

\section{A. System Model}

A parallel relay-assisted FSO communication system is considered, using intensity-modulated direct detection (IM/DD) employing BPSK-SIM. The same modulated information transmitted from the transmitting node to the receiving node indirectly with the help of parallel relays by using two hops. Assume that, $N$ parallel relay paths between the transmitter to the receiver and $N$ parallel relays are placed arbitrarily in each corresponding path. The received signals at the $j^{t h}$ hop in the $i^{t h}$ path $y_{i, j}$ can be modeled as [10]

$$
y_{i, j}=\gamma h_{i, j} x_{i, j}+n_{i, j}, i \in\{1,2, \ldots \ldots N\}, j \in\{1,2\}
$$

where $x_{i, j}$ is the transmitted signals of the $j^{\text {th }}$ hop in the $i^{\text {th }}$ path, $\gamma$ is the detector responsivity, $h_{i, j}$ is the channel fading in the $j^{\text {th }}$ hop in the $i^{\text {th }}$ path, $n_{i, j}$ is signal independent additive white Gaussian noise of the $j^{\text {th }}$ hop in the $i^{t h}$ path with variance $\sigma_{n}^{2}$. As illustrated from Fig. 1 [10], the irradiance ' $h i, j$ ' models the optical intensity fluctuations resulting from atmospheric loss, turbulence and fading can be described as [10]

$$
h_{i, j}=h_{l_{i, j}} h_{s_{i, j}} h_{p_{i, j}}
$$

where $h_{l_{i, j}}$ is the attenuation due to beam extinction and path loss, $h_{s_{i, j}}$ due to scintillation effects and $h_{p_{i, j}}$ due to the geometric spread and pointing errors. 


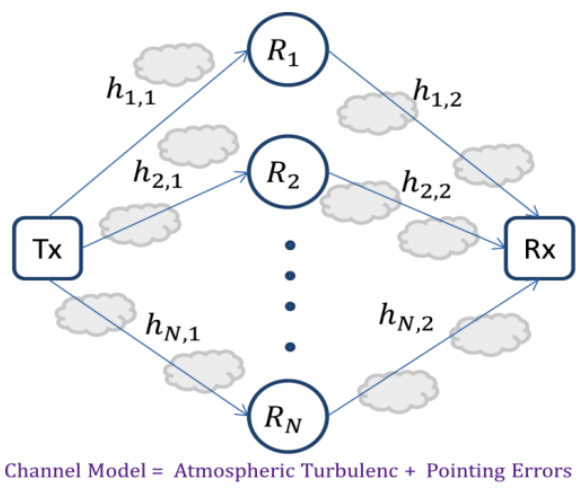

Fig. 1. Parallel relay assisted FSO system.

\section{B. Channel Model}

The atmospheric path loss is modeled by Beers-Lambert law is given as [11]

$$
h_{l_{i, j}}=e^{-\sigma L_{i, j}}
$$

where $\sigma$ is the attenuation coefficient and $L_{i, j}$ is the propagation distance of $j^{\text {th }}$ hop in the $i^{\text {th }}$ path.

In this article, we considered the strong atmospheric turbulence. It can be modeled by gamma-gamma distribution with scintillation parameters $\alpha$ and $\beta$, which are indicated as functions of the Rytov variance and a geometry factor. The probability density function (PDF) of the strong atmospheric turbulence can be modeled as a gamma-gamma distribution is given by [11]

$f_{h_{s_{i, j}}}\left(h_{s_{i, j}}\right)=\frac{2(\alpha \beta)^{(\alpha+\beta) / 2}}{\Gamma(\alpha) \Gamma(\beta)} h_{s_{i, j}}^{(\alpha+\beta) / 2-1} K_{(\alpha-\beta)}\left(2 \sqrt{\alpha \beta h_{s_{i, j}}}\right)$

where $\alpha$ and $\beta$ are the effective number of large and small scale turbulent eddies, $\Gamma(\cdot)$ is the gamma function. $K_{(\alpha-\beta)}$ is the modified Bessel function of the second kind of order $(\alpha-\beta)$.

The misalignment between the transmitter and receiver degrades the performance and reliability of the FSO communication system. By considering a circular detection aperture of radius $r$ and a Gaussian beam, the PDF of $h_{p}$ is given by [12]

$$
f_{h_{p}}\left(h_{p}\right)=\frac{\xi^{2}}{A_{0} \xi^{2}} h_{p}^{\xi^{2}-1}, 0 \leq h_{p} \leq A_{0}
$$

where $A_{0}=[\operatorname{erf}(v)]^{2}$ is the fraction of the collected power at $r=0$. The Gauss error function $\operatorname{erf}(\cdot)$ is defined as $\operatorname{erf}(x)=\frac{2}{\sqrt{\pi}} \int_{0}^{x} e^{-t^{2}} d t$. The radial distance is denoted as $\mathrm{r}$ and $\xi$ is the ratio between the equivalent beam radius at the receiver and the pointing error displacement (jitter) standard deviation at the receiver.

The combined channel distribution for strong atmospheric turbulence regime is given by [13]

$$
\begin{gathered}
f_{h_{i, j}}\left(h_{i, j}\right)=\frac{2 \xi^{2}(\alpha \beta)^{(\alpha+\beta) / 2}}{\left(A_{0} h_{l_{i, j}}\right)^{\xi^{2}} \Gamma(\alpha) \Gamma(\beta)} h_{i, j}^{\xi^{2}-1} \times \\
\int_{h_{i, j} / A_{0} h_{l_{i, j}}}^{\infty} h_{s_{i, j}}^{(\alpha+\beta) / 2-1-\xi^{2}} K_{(\alpha-\beta)}\left(2 \sqrt{\alpha \beta h_{s_{i, j}}}\right) d h_{s_{i, j}}
\end{gathered}
$$

where $\alpha$ and $\beta$ are the effective number of large and small scale turbulent eddies, $\Gamma(\cdot)$ is the gamma function, $K_{(\alpha-\beta)}$ is the modified Bessel function of the second kind of order $(\alpha-\beta)$. The effective number of large and small scale turbulent eddies $\alpha$ and $\beta$ for a spherical wave are given by [11],

$$
\begin{aligned}
& \alpha=\left[\exp \left(\frac{0.49 \delta_{n}^{2}}{\left(1+0.18 d^{2}+0.56 \delta_{n}^{12 / 15}\right)^{7 / 6}}\right)-1\right]^{-1} \\
& \beta=\left[\exp \left(\frac{0.51 \delta_{n}^{2}\left(1+0.69 \delta_{n}^{12 / 15}\right)^{-5 / 6}}{\left(1+0.9 d^{2}+0.62 d^{2} \delta_{n}^{12 / 15}\right)^{5 / 6}}\right)-1\right]^{-1}
\end{aligned}
$$

where $d=\sqrt{k D^{2} / 4 L}, k=2 \pi / \lambda$, is the optical wave number, with $\lambda$ being the operational, $L$ is the length of the optical link and $D$ is the receiver's aperture diameter. The parameter $\delta_{\mathrm{n}}^{2}$ is the Rytov variance and is given as, $\delta_{n}^{2}=$ $0.5 C_{n}^{2} k^{7 / 6} L^{11 / 6}$ and the $C_{n}^{2}$ represents the refractive index structure parameter.

The modified Bessel function $K_{(\alpha-\beta)}$ is expressed in terms of Meijer G function [14, Eq. (14)] and simplified using [15, Eq. (07.34.21.0085.01)], a closed-form expression for the channel model is obtained as [16]

$$
f_{h_{i, j}}\left(h_{i, j}\right)=\frac{\alpha \beta \xi^{2}}{A_{0} h_{l_{i, j}} \Gamma(\alpha) \Gamma(\beta)} G_{1,3}^{3,0}\left[\left.\frac{\alpha \beta h_{i, j}}{A_{0} h_{l_{i, j}}}\right|_{\xi^{2}-1, \alpha-1, \beta-1} \xi^{2}\right]
$$

where $\alpha$ and $\beta$ represent the effective number of large and small scale turbulent eddies, $\Gamma(\cdot)$ is the gamma function.

\section{OUTAGE ANALYSIS}

In the receiver, the received signal should have a minimum SNR to maintain a satisfactory quality of service is called threshold SNR. The outage probability is the probability that the end-to-end output SNR drops less than a specified threshold. The outage probability of the $j^{\text {th }}$ hop in the $i^{\text {th }}$ path can be expressed as

$$
P_{\text {out }_{i, j}}=\operatorname{Pr}\left(\operatorname{SNR}\left(h_{i, j}\right)<S N R_{t h}\right)
$$

where $S N R_{t h}$ is the threshold $S N R$.

The electrical $S N R$ at the receiver can be expressed as,

$$
\operatorname{SNR}\left(h_{i, j}\right)=\frac{\left(\gamma h_{i, j}\right)^{2}}{2 \sigma_{n}^{2}}
$$

To substitute Eqn. (11) in (10), we obtain the outage probability as

$$
P_{\text {out }_{i, j}}=\operatorname{Pr}\left(h_{i, j}<\sqrt{\frac{S N R_{t h} 2 \sigma_{n}^{2}}{\gamma^{2}}}\right)=F_{h_{i, j}}\left(\sqrt{\frac{S N R_{t h} 2 \sigma^{2}}{\gamma^{2}}}\right)
$$

The cumulative distribution function (CDF) of strong atmospheric turbulence channel with pointing error can be defined as

$$
F_{h_{i, j}}\left(h_{i, j}\right)=\int_{0}^{h_{i, j}} f_{h_{i, j}}\left(h_{i, j}\right) d h_{i, j}
$$


The integral can be solved by using the Meijer $G$ function, can be calculated from [17]

$$
F_{h_{i, j}}\left(h_{i, j}\right)=\frac{\xi^{2}}{\Gamma(\alpha) \Gamma(\beta)} G_{2,4}^{3,1}\left[\left.\frac{\alpha \beta}{A_{0} h_{l_{i, j}}} h_{i, j}\right|_{\xi^{2}, \alpha, \beta, 0} ^{1, \xi^{2}+1}\right]
$$

By using Eqn. (8) in (6), the outage probability can be expressed as

$$
P_{\text {out }_{i, j}}=\frac{\xi^{2}}{\Gamma(\alpha) \Gamma(\beta)} G_{2,4}^{3,1}\left[\left.\frac{\alpha \beta}{A_{0} h_{l_{i, j}}} \sqrt{\frac{2 \sigma_{n}^{2} S N R_{t h}}{\gamma^{2}}}\right|_{\xi^{2}, \alpha, \beta, 0} ^{1, \xi^{2}+1}\right]
$$

On parallel relay transmission, the outage performance of each path depends on the outage probability of each hop in the corresponding path. The outage probability of $i^{t h}$ path can be written as

$$
P_{\text {out }_{i}}=1-\prod_{j=1}^{2}\left[1-P_{\text {out }_{i, j}}\right]
$$

The end to end outage probability is obtained as

$$
P_{\text {out }}=\prod_{i=1}^{N} P_{\text {out }_{i}}=\prod_{i=1}^{N}\left\{1-\prod_{j=1}^{2}\left[1-P_{\text {out }_{i, j}}\right]\right\}
$$

\section{NUMERICAL RESULTS AND DISCUSSIONS}

The certain numerical results based on the analytical expressions derived in Section III are presented in this section. The following FSO system parameters are considered in the numerical evaluations. Noise standard deviation $\sigma_{n}=$ $10^{-7} \mathrm{~A} / \mathrm{Hz}$, photo detector responsivity $\gamma=0.5 \mathrm{~A} / \mathrm{W}$, beam radius $w_{L} \cong 2.5 \mathrm{~m}$ at $1 \mathrm{~km}$ distance and jitter standard deviation $\sigma_{s} \cong 30 \mathrm{~cm}$.

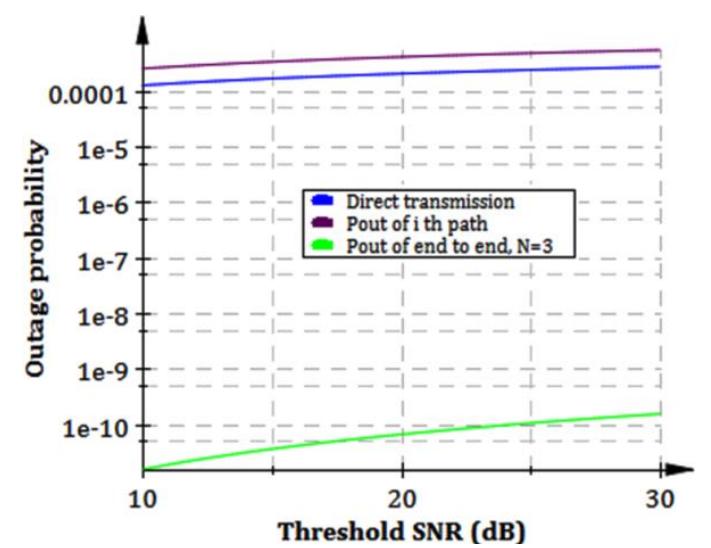

Fig. 2. Outage probability against threshold SNR for FSO system with and without parallel relay.

Fig. 2 depicts the outage performance comparison between the direct transmission and relay assisted FSO system. Two hops and three relays (paths) are considered for the analysis. It can be noticed from Fig. 2, the outage probability of an FSO system is improved through the parallel relay transmission compared to the direct transmission and single path transmission.

The end to end outage probability performance of parallel relay assisted FSO system for the various values of $\alpha=4.2,1$ and $\beta=1$ shown in Fig. 3. The values of $\alpha$ and $\beta$ are the effective number of large and small scale turbulent eddies used to represent the moderate and strong turbulences. It is observed that from the Fig. 3, the outage performance of the system improved along the increasing of $\alpha$ and $\beta$.

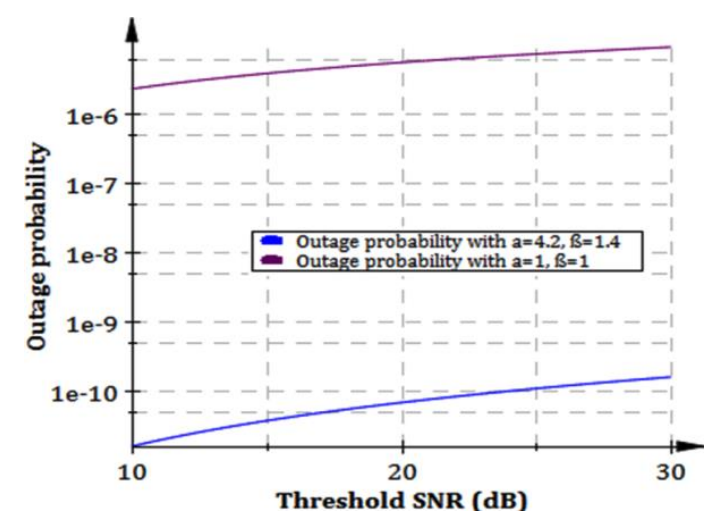

Fig. 3. Outage probability against threshold SNR for parallel relay FSO system with $\alpha=4.2, \beta=1.4$ and $\alpha=\beta=1$.

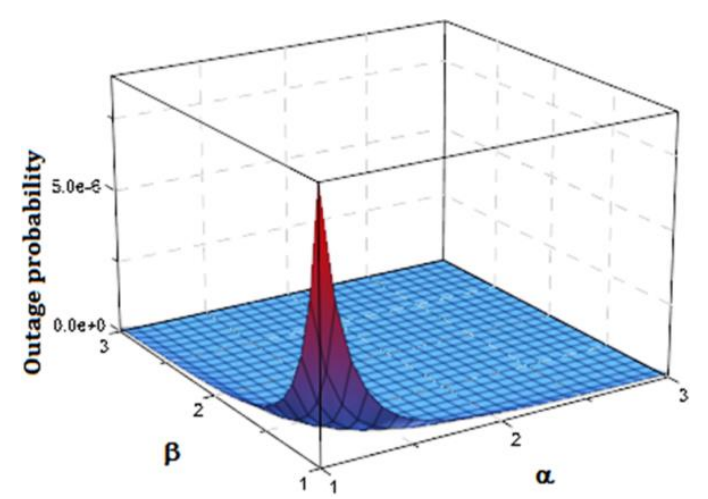

Fig. 4. 3D plot for outage probability variation with respect to $\alpha$ and $\beta$ for parallel relay FSO system.

The 3D plot shown in Fig. 4 depicts the trade-off between the outage performances against the $\alpha$ and $\beta$ for parallel relay assisted FSO system. The 3D graphs are plotted for the values of $\alpha=\beta=3$. From Fig. 4 , it is inferred that the best error rate performances are achieved for large values of $\alpha=\beta=3$.

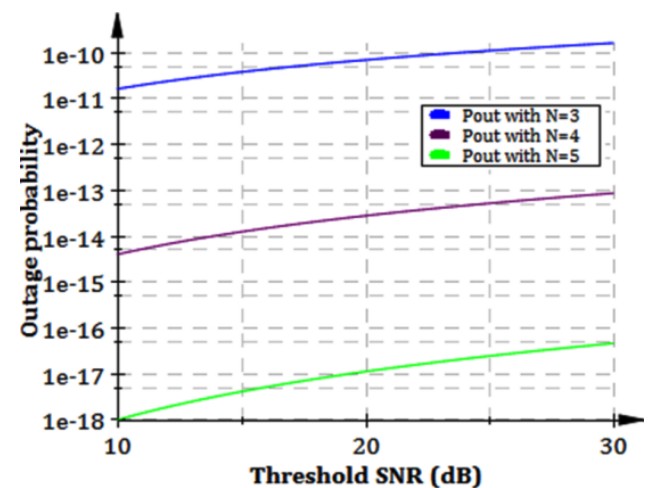

Fig. 5. Outage probability against threshold SNR for parallel relay FSO system with $N=3,4,5$.

Fig. 5 illustrates the outage performance of parallel relay assisted FSO system with various numbers of relay paths. For this analysis 3, 4 and 5 relay paths are considered respectively and each path having two hops. From the figure, it is inferred that the outage performance improves along with the number of relay paths. An FSO system with five parallel relay paths provides the best outage performance compared to the 3 and 4 relay paths. 


\section{CONCLUSION}

The novel exact closed form expressions for the outage probability of BPSK-SIM based parallel relay assisted FSO system over a strong atmospheric channel with pointing error is derived. It is observed that parallel relay assisted FSO system provides the best outage performance compared to the direct transmission. It is also shown that a number of relay paths $(N)$, improves the outage performance. The minimum outage probability obtained for five relay paths $(N=5)$, is $10^{-18}$

\section{REFERENCES}

[1] C. Liu, Y. Yao, Y. Sun, and X. Zhao, "Average capacity for heterodyne FSO communication systems over gamma-gamma turbulence channels with pointing errors," Elect. Lett., vol. 46, pp. 851-853, 2010.

[2] I. B. Djordjevic, "Adaptive modulation and coding for free-space optical channels," IEEE/OSA J. Optical Communications and Net., vol. 2, pp. 221-229, 2010.

[3] S. G. Wilson, M. Brands-Pearce, Q. Cao, and J. J. H. Leveque, "Free space optical MIMO transmission with Q-ary PPM," IEEE Trans. Commun., vol. 53, pp. 1402-1412, 2005.

[4] I. B. Djordjevic, B. Vasic, and M. A. Niefeld, "Multilevel coding in free-space optical MIMO transmission with Q-ary PPM over the atmospheric turbulence channel," IEEE Photon Technology. Lett., vol. 18, pp. 1491-1493, 2006.

[5] H. E. Nistazakis, T. A. Tsiftsis, and G. S. Tombras, "Performance analysis of free-space optical communication systems over atmospheric turbulence channels," IET Communications, vol. 3, no. 8, pp. 1402-1409, 2009.

[6] M. Safari and M. Uysal, "Relay-assisted free-space optical communication," IEEE Trans. Wireless Commun., vol. 7, no. 12, pp. 5441-5449, 2008.

[7] C. K. Datsikas, K. P. Peppas, N. C. Sagias, and G. S. Tombras, "Serial free-space optical relaying communications over gamma-gamma atmospheric turbulence channels," IEEE/OSA J. Optical Communications and Net., vol. 2, no. 8, pp. 576-586, 2010.

[8] M. R. Bhatnagar, "Performance analysis of decode-and-forward relaying in gamma-gamma fading channels," IEEE Photonics Tech. Lett., vol. 24, no. 7, pp. 545-547, 2012.

[9] M. Karimi and M. Nasiri-Kenari, "BER analysis of cooperative systems in free-space optical networks," IEEE/OSA J. Lightwave Tech. vol. 27, no. 24, pp. 5639-5647, 2009.

[10] M. Feng, J. B. Wang, M. Sheng, L. L. Cao, X. X. Xie, and M. Chen, "Outage performance for parallel relay-assisted free-space optical communications in strong turbulence with pointing errors," in Proc. IEEE International Conference on Wireless Communications and Signal Processing (WCSP), 2011, pp. 1-5.

[11] L. C. Andrews and R. L. Phillips, Laser Beam Propagation through Random Media, SPIE Press, vol. 52, 2005.
[12] A. A. Farid and S. Hranilovic, "Outage capacity optimization for free-space optical links with pointing errors," IEEE/OSA J. Lightwave Tech., vol. 25, no. 7, pp. 1702-1710, 2007.

[13] I. E. Lee, Z. Ghassemlooy, W. P. Ng, and M. Uysal, "Performance analysis of free space optical links over turbulence and misalignment induced fading channels," in Proc. IEEE 8th International Symposium on Communication Systems, Networks \& Digital Signal Processing, 2012, pp. 1-6.

[14] V. S. Adamchik and O. I. Marichev, "The algorithm for calculating integrals of hypergeometric type functions and its realization in REDUCE system," in Proc. the International Symposium on Symbolic and Algebraic Computation, pp. 212-224, 1990.

[15] The wolfram functions site. (2001). [Online]. Available: http://functions.wolfram.com/

[16] K. Prabu, S. Bose, and D. S. Kumar, "BPSK based subcarrier intensity modulated free space optical system in combined strong atmospheric turbulence," Optics Communications, 2013.

[17] G. H. Sandalidis, T. A. Tsiftsis, and G. K. Karagiannidis, "Optical wireless communications with heterodyne detection over turbulence channels with pointing errors," IEEE/OSA J. Lightwave Tech., vol. 27 , no. 20 , pp. $4440-4445,2009$.

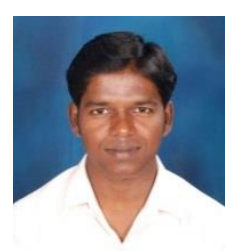

K. Prabu received his bachelor degree in electronics and communication engineering and the master of engineering degree in communication and networking from Anna University, Chennai, India, in 2007 and 2010 respectively. Currently he is pursuing his Ph.D. in free space optical communication in the Department of Electronics and Communication Engineering, National Institute of Technology (NIT), Tiruchirappalli, India. $\mathrm{He}$ is a recipient of Technical Education Quality Improvement Programme (TEQIP) Scholarship [2012-2015] for his Ph.D program in the Department of Electronics and Communication Engineering, National Institute of Technology (NIT), Tiruchirappalli, India. His research interests include free-space optical communications, wireless communications, multiple-input multiple-output (MIMO) systems, and radio over fiber technology.

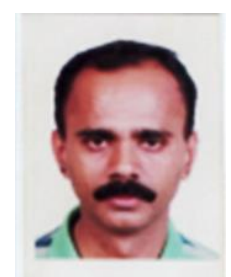

Sriram Kumar received his bachelor degree in electronics and communication engineering from Madurai Kamaraj University, India in 1991 and the master of engineering degree in microwave and optical engineering, from A.C. College of Engineering and Technology, Karaikudi, India, in 1994. He received the $\mathrm{Ph} . \mathrm{D}$. degree in electronics and communication engineering from the Bharathidasan University, India, in 2009. He is currently working as an associate professor in the Department of Electronics and Communication Engineering, National Institute of Technology (NIT), Tiruchirappalli, India. His current research interests include optical networks, smart antennas, optical communications, and microwave integrated circuits. 